\title{
Kernos
}

Revue internationale et pluridisciplinaire de religion grecque antique

$29 \mid 2016$

Varia

\section{Egyptian Gods in the Hellenistic and Roman Mediterranean: Image and Reality Between Local and Global}

\section{Stefano Caneva}

\section{(2) OpenEdition}

12 Journals

\section{Édition électronique}

URL : http://journals.openedition.org/kernos/2437

DOI : $10.4000 /$ kernos. 2437

ISSN : 2034-7871

\section{Éditeur}

Centre international d'étude de la religion grecque antique

\section{Édition imprimée}

Date de publication : 1 octobre 2016

Pagination : 451-456

ISSN : 0776-3824

\section{Référence électronique}

Stefano Caneva, «Egyptian Gods in the Hellenistic and Roman Mediterranean: Image and Reality Between Local and Global », Kernos [En ligne], 29 | 2016, mis en ligne le 01 octobre 2016, consulté le 17 novembre 2020. URL : http://journals.openedition.org/kernos/2437 ; DOI : https://doi.org/10.4000/ kernos. 2437

Ce document a été généré automatiquement le 17 novembre 2020.

Kernos 


\title{
Egyptian Gods in the Hellenistic and Roman Mediterranean: Image and Reality Between Local and Global
}

\author{
Stefano Caneva
}

\section{RÉFÉRENCE}

Laurent BRICAULT, Miguel John VERSLUYS (éd.), Egyptian Gods in the Hellenistic and Roman Mediterranean: Image and Reality Between Local and Global, Caltanissetta, Salvatore Sciascia Editore, 2012.1 vol. $17 \times 24 \mathrm{~cm}$, viii+144 p. (Supplemento a Mythos, 3). ISBN :

978-88-8241-418-4.

1 Le troisième Supplément de Mythos a été animé par le groupe de recherche international sur les "études isiaques" et apporte une contribution variée à la discipline, à travers cinq cas d'étude ciblés et une mise au point transversale de l'état de l'art, offrant une panoramique dense des quinze ans d'activité de ce groupe international et des priorités dans la définition des perspectives d'avenir.

2 Les éditeurs ont décidé d'assortir les quatre articles qui constituent le cœur de la publication, issus d'une journée d'études des doctorants tenue à Leyde le 26 janvier 2011, de deux contributions de grande envergure méthodologique et programmatique (Bricault/Veymiers; Versluys). Celles-ci se penchent respectivement sur la dimension historiographique et théorique de l'étude de la diffusion des divinités égyptiennes de la famille isiaque dans la Méditerranée hellénistique et romaine. Cette décision éditoriale relève de la conscience, par la génération qui guide à présent ce courant d'étude, de l'importance de nourrir le réseau international qu'ils ont établi au cours des deux dernières décennies. Cette conscience apparaît comme prioritaire d'un point de vue aussi bien humain que méthodologique, en considération de la valeur essentielle de la confrontation internationale dans la maturation scientifique des jeunes chercheurs (p. 2-3), mais aussi parce que cette collaboration élargie et continue est porteuse d'une 
approche inclusive par rapport aux différentes tendances et perspectives en vogue dans les courants académiques locaux ( $\mathrm{p}$. vii).

3 Comme le montre le titre, la perspective unifiant (dans la variété) le volume consiste dans la mise en exergue des traits de continuité et de différentiation qui caractérisent la diffusion et l'enracinement des cultes des divinités égyptiennes dans le bassin méditerranéen ancien. Les deux axes d'analyse permettant d'ordonner et de valoriser ces données variées sont le temps (continuité et rupture au passage de la période hellénistique à la période romaine) et l'espace (transversalité géographique et spécificités régionales). L'introduction historiographique de Bricault/Veymiers assure que cette disposition n'est pas un artifice visant à unifier des contributions disparates, mais une exigence de méthode inhérente à l'évolution des études. Ainsi, la bibliographique offre une occasion de recontextualiser les études de référence de la discipline par rapport aux différentes paradigmes théoriques de son histoire. Pour se limiter à deux cas, l'horizon historiographique du fondamental Recueil des Inscriptions concernant les cultes isiaques (2005) renvoie à une période où l'intérêt principal des études isiaques se portait sur la « compréhension des cultes dévolus à la gens isiaque en dehors d'Égypte ", ce qui fait de la constitution d'un répertoire concernant l'Égypte (et ses langues) une réelle urgence (p. 5-6). De manière similaire, l'augmentation rapide de la documentation, ainsi que la mise à jour des questionnements et des méthodes de recherche, imposent de reconsidérer le balancement entre la collection de répertoires régionaux et les interprétations d'ensemble. Aujourd'hui, la dimension globale de la Méditerranée, sur laquelle était centrée l'initiative éditoriale des EPRO, apparaît comme une indication des limites de pertinence de la discipline plutôt que comme échelle dominant l'interprétation, qui devrait se positionner davantage au niveau régional ou même de sites individuels (p.12). Plus généralement, la contribution de Bricault/ Veymiers a le mérite de fournir une topographie des dossiers, des disciplines et même des catégories de support archéologique nécessaires pour placer les résultats des études déjà menées dans un cadre plus homogène, favorisant la comparaison intermédiale ainsi que synchro- et diachronique. Le besoin de programmer le passage des répertoires isiaques aux plateformes numériques n'est pas moins urgent: la multiplication, la standardisation et la mécanisation des liens entre des dossiers différents apporteraient sans doute une contribution fondamentale à la recherche ainsi qu'à la didactique de la discipline.

Ce vaste bilan bibliographique, qui combine la chaleur humaine du portrait de famille avec une synthèse limpide des desiderata pour la recherche à venir, est intégré par l'analyse théorique menée par Versluys, qui se pose l'objectif ambitieux de développer et de tester de nouvelles questions pour interroger le dossier isiaque. Cette initiative répond au constat du dépassement de deux catégories cumontiennes qui ont longtemps guidé les études : celles de « religions orientales » et de « diffusion ». La déconstruction de ces deux catégories conduit à abandonner une définition de l'objet de la discipline comme "something genuinely Egyptian/Oriental diffused from a Nilotic core to a Mediterranean periphery» (p.28). Par ailleurs, une nouvelle piste interprétative ne devrait pas se limiter à une étude complètement décentralisée des processus d'appropriation des cultes isiaques dans des contextes locaux limités. En effet, cette approche ne permettrait pas de comprendre pourquoi, dans tous ces contextes, les cultes égyptiens liés à la famille isiaque étaient de fait perçus comme une catégorie unitaire et reconnaissable. 
5 Versluys propose une solution possible pour la compréhension à la fois locale et globale, variée et unitaire des attestations cultu(r)elles de la gens isiaque dans le patrimoine symbolique que ces divinités portaient avec elles en tant qu'égyptiennes. Le point de départ se situe dans une reconsidération, dans une nouvelle perspective d'histoire culturelle, de la catégorie du "mirage égyptien» en général, et plus précisément de la thèse bien connue de Momigliano dans son ouvrage Alien Wisdom: notamment le fait que l'obscurité de la culture égyptienne aurait empêché un véritable contact culturel avec les Grecs, qui se limitèrent à considérer l'Égypte comme un " repository of unusual knowledge $»^{1}$ placé dans une dimension d'immobilité hors du temps, plutôt que comme une réelle entité politique et culturelle. Or, Versluys prend justement ses distances avec une réutilisation littérale de la thèse de Momigliano, dont la nature rétrospective, simplificatrice et politisée a été dévoilée et rejetée par une mise à jour des dossiers et de la méthodologie de recherche sur les processus d'interaction culturelle dans le monde gréco-romain ${ }^{2}$. Versluys s'appuie plutôt sur une idée plus nuancée, déjà bien attestée chez les Grecs pré-hellénistiques et remise en vogue par Froidefond à la même période que les travaux de Momigliano $^{3}$ : il s'agit d'une vision grecque de la culture égyptienne comme investie d'un fort capital symbolique et donc capable de solliciter la réflexivité des Grecs sur ses propres schémas culturels et politiques.

6 Cette Égypte «bonne à penser» offre à Versluys la solution méthodologique pour thématiser le fond cultuel et culturel commun de la réception et l'appropriation des divinités de la famille isiaque dans le monde méditerranéen. Le traitement du cas d'étude choisi, celui de la construction de la figure d'Antinoos à la fois dans la perspective locale de la fondation d'Antinopolis et dans celle, globale, du projet de renaissance culturelle d'Hadrien, met bien en valeur la puissance de la figure culturelle de l'Égypte en tant que terre religieuse. Dans l'interprétation de Versluys, cette caractérisation offrirait le pendant, dans la dimension cultuelle, au choix qu'Hadrien fit de la Grèce en tant que lieu agrégateur de l'identité culturelle de l'Empire. La perspective ouverte par Versluys mérite d'être prise en compte dans d'autres cas d'études (comme le fait en effet Bülow Clausen dans ce volume), bien que sa validité paraisse pertinente pour la seule époque romaine plutôt que pour une analyse embrassant aussi la période hellénistique. Il faudra rappeler que Froidefond conçut l'idée du mirage égyptien en travaillant sur les sources littéraires de la Grèce classique. De surcroît, les études isiaques ont reconnu depuis longtemps le rôle crucial joué par le royaume des Ptolémées en tant que plaque tournante de l'interaction constante de l'Égypte avec le bassin méditerranéen. ${ }^{4}$ Le mirage se placerait dès lors avant et après la période hellénistique, c'est-à-dire lorsque les Grecs (et les Romains ensuite) ne se trouvèrent qu'en contact éloigné ou occasionnel avec les vrais porteurs de la culture égyptienne. Il semble révélateur, à ce propos, qu'en attribuant « some kind of agency of its own» à l'Égypte en tant que symbole culturel (p.35), Versluys ne prenne pas en considération le rôle de ceux qui détenaient le contrôle de cette tradition: les prêtres égyptiens avec leurs propres agendas, les premiers auteurs de la construction du mythe d'Antinoos avant que les mécanismes et les vecteurs de la propagande d'Hadrien ne le replacent dans la totalité de l'Empire et ne le re-fonctionnalisent pour une variété d'acteurs et d'applications locaux. Le cœur du problème est le fait que la dimension culturelle du mirage égyptien ne se pose pas en amont, mais comme conséquence de ses aspects socio-politiques : c'est la transformation de l'Égypte en province romaine et, par conséquent, la limitation de l' "agentivité » de son élite à un niveau local, qui 
justifie la nécessité, pour les messages culturels issus de l'Égypte, de rejoindre le cœur de l'Empire gréco-romain par le biais de médiateurs, qui s'approprient son pouvoir symbolique et le manipulent pour leurs propres besoins.

7 Les perspectives mises en exergue dans les contributions d'ouverture sont appliquées dans les analyses de cas d'étude qui les suivent. Matricon-Thomas discute les dynamiques sociales et religieuses de l'enracinement du culte d'Isis à Athènes depuis la fin du Iv siècle av. J.-C. jusqu'à l'antiquité tardive, en se concentrant en particulier sur deux questions: le changement, à travers le temps, du profil social des acteurs s'engageant dans la pratique et la promotion des cultes pour Isis, et l'évolution diachronique des rapports entre cette divinité et le panthéon local de la cité. En ce qui concerne le premier point, l'A. offre un panorama diachronique des étapes, ou vagues, du succès des cultes isiaques en attirant l'attention sur la variété de ses acteurs (institutionnels, individuels, confédérés en groupes professionnels ou d'intérêt), de facteurs (intérêts cultuels, propagande, processus de hiérarchisation) et de dynamiques d'interaction entre la dimension locale et la politique étrangère, avec une attention particulière pour les moments où une proximité plus accentuée avec les Ptolémées a pu favoriser le développement d'une piste isiaque dans les stratégies de la polis ou de membres de son élite. Le problème de genre des acteurs des cultes est aussi pris en considération par Matricon-Thomas, sans pourtant que la composition de la documentation ne permette de trancher la question de savoir si la concentration majeure d'acteurs féminins dépend de causes rituelles ou de représentations sociales. En ce qui concerne l'évolution cultuelle de la figure d'Isis, le parcours offert par l'A. nous montre l'ascension, depuis la fin du $\mathrm{II}^{\mathrm{e}}$ et pendant le $\mathrm{I}^{\mathrm{er}}$ siècle av. J.-C., de la déesse par rapport à Sarapis, un changement renversant le rapport signalé à l'intérieur du couple divin dans la documentation des garnisons militaires du $\mathrm{III}^{\mathrm{e}} \mathrm{s}$. Un aspect typiquement (bien que non exclusivement) athénien se signale dans le lien spécial entre la déesse et Déméter qui en Attique relève de l'influence des cultes éleusiniens. Ces rapports, tracés à travers les pratiques cultuelles ainsi qu'à travers l'iconographie, offrent un cadre fascinant bien que nécessairement lacunaire en raison de l'état des sources. On regrette seulement qu'à la fin de la contribution, l'A. ait abandonné les outils sociologiques employés pendant la discussion pour présenter une évaluation générique du succès du culte d'Isis par rapport à sa capacité de " toucher les Athéniens dans leurs religiosité intime» (p.55). Cette polarisation, conceptuellement dépassée, sur l'opposition entre raisons spirituelles et " profanes » du succès des cultes égyptiens ne rend pas suffisamment hommage aux efforts méthodologiques déployés dans la contribution et n'ajoute rien à ses intéressantes conclusions.

8 La contribution de Nagel déplace l'analyse dans le Nord de l'Afrique pour délimiter une fascinante esquisse de comparaison des manifestations des cultes isiaques entre les régions touchées par des influences égyptiennes, phéniciennes (et ptolémaïques) au cour du $\mathrm{I}^{\mathrm{er}}$ millénaire, et celle où ces cultes s'installèrent, ou furent réorganisés, sous domination romaine. En combinant l'analyse des types iconographiques et des sources textuelles, Nagel montre que les régions où la figure d'Isis connut une longue période d'intégration dans les traditions locales pré-romaines, comme en Cyrénaïque, Tripolitaine et Africa Proconsularis, les effets de la «romanisation » des cultes isiaques furent relativement limités et superficiels. Par contre, l'entrée tardive des régions de l'Afrique nord-occidentale (Numidie et Maurétanie) dans une koiné culturelle et politique méditerranéenne se signale, par rapport au sujet qui nous intéresse, dans un 
impact plus fort des formes romanisées du culte de Sérapis et Isis, qui s'installèrent dans ces régions en particulier pendant le $\mathrm{II}^{\mathrm{e}}$ et le $\mathrm{III}^{\mathrm{e}} \mathrm{s}$. ap. J.-C. L'A. indique les traits caractérisant cette tendance dans le succès particulier de Sérapis, surtout à l'initiative des membres de l'élite administrative et de l'armée; dans la forte caractérisation romaine du dieu dans l'iconographie, les épithètes et l'association fréquente avec l'empereur; dans le rôle marginal des synnaoi theoi de la famille isiaque, beaucoup plus important dans les régions de longue fréquentation isiaque. Le cadre se complète par l'analyse des cas de Carthage et de Iol Caesarea, dont la spécificité se signale pour deux raisons opposées, mais unifiées par l'influence du pouvoir politique: dans le cas de Carthage, la destruction de la ville marqua une rupture profonde entre la première pénétration de la figure d'Isis dans la culture punique et le nouveau succès de ces cultes à la période romaine ; par contre, la perméabilité réduite de Iol Caesarea aux modèles d'importation romaine, un facteur marquant une discontinuité avec le reste de la Mauretania Caesarensis, se justifie en relation avec le fort soutien donné à l'imagerie isiaque par le projet idéologique de Juba II et de Cléopâtre Sélénè, fille de Cléopâtre VII.

Après l'échelle civique et macro-régionale, l'analyse se concentre, dans l'étude de Bülow Clausen, sur un site spécifique, le sanctuaire d'Isis à Beneventum. La portée des propositions de l'A. se déploie toutefois dans la dimension plus vaste de l'idéologie du temps de Domitien, qui fit de la ville un lieu de mémoire du succès de l'empereur et de sa famille. En ce qui concerne strictement la question des relations entre les échelles locale et globale dans la caractérisation des cultes isiaques, Bülow Clausen accepte le défi posé par un dossier particulièrement complexe comme celui des fragments statuaires provenant de Bénévent. Faute de fouilles systématiques, ceux-ci ont toujours été classés selon un critère aprioristique : l'appartenance à l'Iseum a été fondée sur la nature égyptienne ou égyptisante de certains fragments, tandis que tout ce qui est plus proprement gréco-romain ne ferait pas partie du projet architectural du sanctuaire isiaque. Même si l'absence de certitude par rapport au contexte original ne permet pas à l'A. d'atteindre des conclusions sur la présence d'éléments gréco-romains en interaction avec les égyptiens dans l'Iseum, la portée méthodologique de la contribution demeure néanmoins forte. Les fragments sculpturaux de Bénévent sont intégrés dans un réseau interprétatif qui, du sanctuaire, s'élargit à la cité et, de la cité, au cadre comparé de Pompéi et de Rome. Par le biais des projets des Flaviens, la configuration des rapports entre traditions égyptiennes et gréco-romaines finit par situer symboliquement l'Iseum à la rencontre entre la propagande de l'empereur et une Égypte qui, selon le cadre théorique développé par Versluys en ouverture, se configure comme chargé d'un fort capital symbolique quand il est regardé du côté gréco-romain de l'Empire.

Dans la dernière contribution, Casaux propose encore une autre configuration de l'axe spatial d'analyse proposé par les éditeurs, en le déplaçant cette fois sur le plan conceptuel de la construction d'une altérité orientale par Tertullien. Le rapport entre spécificité et globalité est dès lors décliné dans la perspective du positionnement critique des religions autres que la religion chrétienne dans la polémique de l'apologète. D'un côté, Casaux montre que Tertullien, non seulement, ne sent pas le besoin de développer une argumentation différente pour chaque "culte oriental", mais que la catégorie unitaire d'imitatio diabolica, permettant de refuser tout compétiteur de la vraie religion, s'applique de la même façon aux hérésies qui menacent l'unité de l'Église. Cette thèse est confirmée par l'analyse d'un ouvrage tardif et peu étudié de Tertullien, De ieiunio adversus Psychicos, qui constitue aussi la section la 
plus innovante de la contribution de Casaux. Dans ce cas, Tertullien, ayant embrassé le montanisme, se montre prêt à appliquer le modèle de l'imitatio diabolica au parti chrétien qu'il a abandonné. Les parallèles polémiques établis avec les cultes orientaux suivent ce changement de front, en devenant désormais des preuves de la proximité de toute fausse foi avec l'inspiration de l'Ennemi, contre la vérité divine unique dans laquelle se reconnaît Tertullien.

11 Qu' y a-t-il de spécifique dans les cultes des divinités égyptiennes dans le monde méditerranéen, aux périodes hellénistique et romaine? Ce petit volume apporte une contribution variée et inspirante à ce vaste questionnement, qui semble caractériser la nouvelle phase d'étude délimitée par Bricault, Veymiers et Versluys. Comme le souligne ce dernier, le dépassement d'une interprétation linéaire (départ d'un centre égyptien et diffusion vers des périphéries méditerranéennes) force à développer d'autres perspectives de travail. D'ailleurs, le succès des études isiaques et leur positionnement de plus en plus transversal dans des axes de recherche concernant l'histoire du monde gréco-romain pose nécessairement la question théorique de la pertinence d'une catégorie scientifique de "cultes isiaques» comme discipline autonome dans les recherches sur les polythéismes du monde hellénistique et romain. D'un côté, les outils méthodologiques, d'ordre religieux et sociologique, concernant l'étude diachronique des panthéons locaux dans une perspective comparatiste, la large utilisation d'approches interdisciplinaires, l'urgence du renouvellement technologique dans la gestion de dossiers documentaires en croissance continue, sont des facteurs qui renvoient à une appartenance des cultes isiaques au plus large monde des interactions entre les systèmes polythéistes de la Méditerranée ancienne. D'autre part, il y a en effet au moins une dimension spécifique des cultes pour la famille isiaque : c'est qu'ils sont égyptiens, et que cette origine ne comporte pas seulement une caractérisation particulière de la figure de ces divinités et des manières d'interagir avec eux, mais aussi un capital symbolique bien mis en évidence par Versluys par rapport à la construction de messages culturels et idéologiques par les non Égyptiens.

Et pourtant, je ne suis pas entièrement convaincu que le mirage égyptien puisse offrir la catégorie qui permette d'embrasser la totalité des périodes et des régions ciblées par la discipline. Le risque évident est de retourner à une étude des dieux égyptiens en dehors de l'Égypte. Pour charger notre analyse d'une puissance herméneutique véritablement inclusive, il faudra soit substituer à la catégorie de "mirage " un autre concept opératoire, soit réinterpréter le «mirage» dans une perspective dynamique, qui n'était pas présente dans les études de Froidefond et de Momigliano, mais qui a commencé à émerger dans l'analyse de Moyer : à savoir, comme l'interface entre des acteurs égyptiens qui se font codificateurs et émetteurs actifs de leurs traditions pour des étrangers, en relation avec leurs propres agendas, et des acteurs méditerranéens (au sens large de Braudel bien entendu) qui eux-mêmes opéreraient à la fois comme récepteurs de ces messages et comme émetteurs de nouvelles variations sur ces thèmes, adaptant ainsi le capital symbolique de l'Égypte aux nécessités d'autres milieux géographiques et socio-culturels. Départ de l'Égypte et retour, pour un nouveau départ : c'est dans ce cercle herméneutique, à mon avis, que se situent l'histoire et le programme des études isiaques. 


\section{NOTES}

1. .A. momigliano, Alien Wisdom: The Limits of Hellenization, Cambridge, 1975, p. 3

2. .En ce qui concerne le panorama culturel dans lequel opéra Momigliano, voir déjà R.K RITNER, «Implicit Models of Cross-Cultural Interaction: A Question of Noses, Soap, and Prejudice », in J.H. JOHNSON (éd.), Life in a Multicultural Society: Egypt from Cambyses to Constantine and Beyond, Chicago, 1992, p. 283-290. Plus récemment, I.S. MOYER, Egypt and the Limits of Hellenism, Cambridge 2011, en particulier 1-36, Introduction: The Absence of Egypt.

3. .C. FROIDEFOND, Le Mirage égyptien dans la littérature grecque d'Homère à Aristote, Paris, 1971.

4. .Voir, par exemple, L. BRICAULT, "La diffusion isiaque : une esquisse ", in P.C. BOL et al. (éd.), Fremdheit - Eigenheit. Ägypten, Griechenland und Rom. Austausch und Verständnis, Stuttgart, 2004, p. 548-556 ; ID., Isis dame des flots, Liège, 2006.

\section{AUTEURS}

\section{STEFANO CANEVA}

Università di Padova - Marie Curie 